\title{
Single-phase-field model of stepped surfaces
}

\author{
M. Castro, ${ }^{1, *}$ A. Hernández-Machado, ${ }^{2}$ and R. Cuerno ${ }^{3}$ \\ ${ }^{1}$ Grupo Interdisciplinar de Sistemas Complejos (GISC) and Grupo de Dinámica No Lineal (DNL), \\ Escuela Técnica Superior de Ingeniería (ICAI), Universidad Pontificia Comillas, E-28015 Madrid, Spain \\ ${ }^{2}$ Departament d'Estructura i Constituents de la Matèria, Universitat de Barcelona, Avinguda Diagonal 647, \\ E-08028 Barcelona, Spain \\ ${ }^{3}$ Departamento de Matemáticas and GISC, Universidad Carlos III de Madrid, Avenida de la Universidad 30, E-28911 Leganés, Spain
}

\begin{abstract}
We formulate a phase-field description of step dynamics on vicinal surfaces that makes use of a single dynamical field, at variance with previous analogous works in which two coupled fields are employed, namely, a phase-field proper plus the physical adatom concentration. Within an asymptotic sharp interface limit, our formulation is shown to retrieve the standard Burton-Cabrera-Frank model in the general case of asymmetric attachment coefficients (Ehrlich-Schwoebel effect). We confirm our analytical results by means of numerical simulations of our phase-field model. Our present formulation seems particularly well adapted to generalization when additional physical fields are required.
\end{abstract}

DOI: 10.1103/PhysRevE.79.021601

PACS number(s): 81.10.Aj, 68.35.Ct, 81.15.Hi, 81.15.Aa

\section{INTRODUCTION}

Crystalline surfaces whose orientation is close to (but does not precisely coincide with) a high symmetry orientation are usually called vicinal surfaces [1]. Their shape basically consists of flat terraces separated by steps of one or a few atomic sizes, which makes them suited for many different applications, such as sites for preferential catalytic activity or as templates for epitaxial growth by, e.g., molecular beam epitaxy (MBE). The most conspicuous example of the latter takes place in the so-called step-flow mode in which deposited adatoms diffuse on the terraces and attach to preexisting steps. In order to gain control on the (technologically desirable) conditions under which the crystals thus grown are smooth and have sharp interfaces, a large effort has been directed to the study of far-from-equilibrium crystal growth phenomena onto this type of surfaces [2,3]. Moreover, also equilibrium crystalline surfaces that are below their roughening temperature $T_{R}$ feature a stepped morphology akin to that of vicinal surfaces, so that the problem of, say, thermal relaxation of a crystalline surface below $T_{R}$ bears conceptual similarities to growth onto vicinal surfaces, and has attracted a similar interest both from the experimental [4] and from the theoretical [5] points of view.

The theoretical study of growth on vicinal surfaces in the step-flow regime originates back in the classic work by Burton, Cabrera, and Frank (BCF) [6]. The realization that many diverse effects can nontrivially modify the simplest solution of a uniformly moving train of straight steps has generated a strong interest in these systems as paradigmatic cases in which nonequilibrium statistical mechanics and nonlinear pattern formation meet [7]. Thus, steps are morphologically unstable to meandering [8] and bunching, and fluctuations in the driving beam flux and island nucleation may induce interface disorder (kinetic roughening). A large variety of morphological behaviors ensues (see, e.g., [9-12]), that indeed

\footnotetext{
*marioc@upcomillas.es
}

correlates with the variety of morphologies that have been found experimentally.

In general one considers a train of steps separated by terraces. On these terraces the adatoms arriving from a vapor can diffuse and eventually impinge towards one step becoming attached to it. Adatom desorption from the terraces can also take place, although MBE conditions frequently correspond to negligible desorption. Subsequent movement of the adatoms along the interface (the step itself) separating the terraces is allowed and is a thermally activated process. The theoretical description of this system has been successfully performed during recent years [9-12] although the interplay among the different mechanisms involved is still unclear. Thus, full understanding has not been achieved yet in the case of the step bunching instability [13], step-step interactions [9], or the so-called kink Ehrlich Schwoebel (KESE) instability [14].

Given that these continuum theories are usually formulated in the form of moving boundary problems (see Sec. II A), their main difficulty lies in the nonlinear implicit coupling between the diffusive dynamics at the terrace and at the curved steps. An important procedure to provide a more compact theoretical description is the derivation of effective interface evolution equations for the step profile. Although highly informative on the system behavior (an overview with references can be found in [15]), these equations are only valid in principle within appropriate asymptotic approximations, and are many times limited, say, to small slope conditions. A more quantitative description of the system-that, e.g., allows us to link continuum model parameters with microscopic features, the study of more general geometries or to explore parameter values outside asymptotic regimesrequires numerical solution of the moving boundary problems. As is usually the case with free and moving interface problems, this faces severe numerical issues related with front tracking. A way to circumvent these is the formulation of a related problem in which the physical concentration field is coupled to an auxiliary phase field that accounts in a natural way for the position of the moving interface as that (thin) area across which the phase field varies appreciably. The 
phase-field method [16] has indeed found great success in the numerical simulation of diverse physical phenomena whose constitutive laws lead to free and moving boundary problems, such as solidification from a melt, various phase transitions such as in liquid crystals, solid-state structural transformations, electrodeposition, grain growth, fracture, etc. [17], to the extent that it is currently considered as a method of choice for multiscale simulations of materials [18].

In the specific case of step dynamics, to date several different phase-field models have been already proposed. In all of them, there is essentially one physical field (namely, the adatom concentration for each terrace), whose dynamics is coupled to that of the phase-field proper. Then, the various formulations can be grouped taking into account whether they consider the same attachment kinetics from both sides of the step [19-21], or else [22-25] they allow for different attachment kinetic coefficients for adatoms approaching the step from the upper and the lower terraces, implementing the Ehrlich-Schwoebel (ES) effect [2,3,8]. We stress that, in all these approaches, at least two independent fields are considered, so that further extensions of the model involving, for instance, diffusion of adatoms along the step would possibly imply the incorporation of yet a new diffusive field at the step, coupled with the other fields in the model.

Alternatively, there have been successful applications of the phase-field approach in which a single field is employed, whose dynamics is actually shown (in the various contexts) to fully reproduce that of the original moving boundary problem. Moreover, the dynamical equation for this field many times resembles quite closely models of critical dynamics for order parameters [26], so that its physical interpretation is usually transparent. Examples can be found in problems such as imbibition [27], viscous flow [28] or heterogeneous nucleation [29] - this being a promising point of departure for further extensions - and other problems as reviewed, e.g., in [30]. The advantages of this approach are clear both conceptually and computationally. From the theoretical point of view, it provides a more compact framework that can always be enlarged by additional fields if the description of new physical effects requires such type of generalization.

In this paper we address the dynamics of steps on vicinal surfaces in the step-flow regime, through this type of singlefield phase-field approach. We consider the standard problem of a single step between two terraces in the general case of asymmetric attachment. This is a reference case considered in many theoretical studies. We show analytically that our phase-field model is equivalent, in the sharp-interface limit, to the classic BCF nonlinear moving-boundary formulation. Moreover, we confirm this conclusion through numerical simulations of our phase-field model, in which we specifically reproduce the basic features of the Bales-Zangwill meandering instability [8] that results from the ES effect.

The paper is organized as follows. In the next section we set up both the moving boundary problem we wish to reproduce, and the single phase-field equation that is equivalent to it. Section III is devoted to proving such an equivalence through an asymptotic study, while these analytical results are checked against direct numerical simulation in Sec. IV. We conclude in Sec. V with a brief discussion and outlook.

\section{FORMULATION OF THE PHASE-FIELD MODEL}

\section{A. Macroscopic model of terrace edges during step flow}

The classic BCF system that provides for us the macroscopic or moving boundary problem of reference consists of a set of coupled equations for the adatom concentration fields, $c_{k}$, on the various terraces composing the vicinal surface. Considering a reference frame comoving along the $z$ direction with the average step velocity $V$, these read as [8]

$$
\begin{gathered}
\partial_{t} c_{k}=D \nabla^{2} c_{k}+F-\frac{c_{k}}{\tau}-V \partial_{z} c_{k}, \\
V_{n}=\nu_{+}\left(c_{k+1}-c_{e q, k}\right)+\nu_{-}\left(c_{k}-c_{e q, k}\right),
\end{gathered}
$$

where

$$
\begin{gathered}
c_{e q, k}=c_{e q}^{0}-\Gamma \kappa_{k}, \\
\frac{V_{n}^{l}}{\Omega}=D \partial_{n} c_{k}+V_{n}^{0} c_{k}-D \partial_{n} c_{k+1}-V_{n}^{0} c_{k+1} .
\end{gathered}
$$

In Eq. (1), $F$ is the deposition flux of atoms, and $\tau$ is the characteristic time for desorption. The combination of Eqs. (2) and (4) provides the boundary conditions, $\Omega$ being the atomic volume. $V_{n}$ is the normal velocity at each point of the terrace edge, that is defined by

$$
V_{n}=V_{n}^{0}+V_{n}^{l}=\frac{V+\partial_{t} h_{k}}{\sqrt{1+\left(\partial_{x} h_{k}\right)^{2}}},
$$

where $h_{k}(x, t)$ is the position of the terrace edge along the $k$ th step coordinate, $x$, at time $t$, and $V_{n}^{l}$ is the normal velocity in the comoving frame. Parameters $\nu_{ \pm}$are the attachment rate contants for adatoms arriving from the upper $(-)$ and lower $(+)$ sides of the step, $c_{e q}^{0}=F \tau$ is the equilibrium value of the concentration at infinity, $\Gamma$ is the surface tension (that will be assumed a constant), and $\kappa_{k}$ is the step curvature,

$$
\kappa_{k}=-\frac{\partial_{x}^{2} h_{k}}{\left[1+\left(\partial_{x} h_{k}\right)^{2}\right]^{3 / 2}} .
$$

As shown by Bales and Zangwill (BZ) [8], Eqs. (1)-(4) predict a meandering instability by which a train of straight steps is morphologically unstable to transverse perturbations. The instability is a consequence of the unbalance of the diffusive flux of adatoms attaching to a step from the two adjacent terraces, when the kinetic attachment coefficients are different, $\nu_{+} \neq \nu_{-}$. Frequently, this occurs as a result of the ES effect, by which adatoms experience a barrier to approaching a step from the upper terrace, due to the loss in coordination [2,3]. Mathematically, considering a single step, it can be seen that, to linear order, periodic perturbations with wave vector $q, h_{q}(t)$, of a straight step evolve in time as $h_{q}(t) \sim e^{\omega_{q} t}$, where the linear dispersion relation has the form (in a long wavelength approximation)

$$
\omega_{k}=a_{2} q^{2}-a_{4} q^{4},
$$

where $a_{2,4}$ are positive constants that depend on the model parameters. This dispersion relation was obtained by BZ in the present context, and turns out to describe the linear re- 
gime for the well-known Kuramoto-Sivashinsky (KS) equation [31]. Actually, in the case of finite desorption that we are considering, $\tau<\infty$, the KS equation has indeed been shown to provide the nonlinear description of a single isolated step [32]. The case of no desorption $\tau \rightarrow \infty$ is more subtle, the shape of the nonlinear evolution equation depending nontrivially on the ES effect being strong [10] or weak [33].

\section{B. Phase-field equations}

We next introduce a one-field phase-field model that contains all the ingredients of the macroscopic model in a simple, compact, and numerically efficient fashion. Moreover, as we will show in Sec. IV we reproduce the linear dispersion relation of the macroscopic theory and, eventually, we can simulate the system in the nonlinear regime. The phase-field model equation is

$$
\partial_{t} \phi=\frac{A-\phi}{\tau}+\nabla \cdot[\Gamma(\phi) \nabla \mu]-\mathbf{E} \cdot \nabla \sigma(\phi),
$$

where $A$ is a constant that takes different values on the two sides of the step, as does the phase field $\phi$ itself thus providing the step position as its zero level set. Moreover, $\phi$ is also related to the value of the macroscopic physical adatom concentration field, in a form to be specified below. The first term on the right-hand side (rhs) of Eq. (8), which accounts for desorption, precisely insures that the order parameter $\phi$ takes different values for the different terraces far from the step. As for the second term on the rhs of Eq. (8), a chemical potential, $\mu$, appears, that is given by

$$
\mu=\frac{\delta \mathcal{F}}{\delta \phi}=-V^{\prime}(\phi)-\epsilon^{2} \nabla^{2} \phi,
$$

where $V(\phi)$ is the potential which determines the local minima of the phase field. Generically, those minima correspond to the (vertical) lattice positions. More specifically, we force the phase field to define the terrace position at equidistant lattice spacings $a$. Thus, we can take, for instance,

$$
V(\phi)=-\cos (2 \pi a \phi),
$$

where $\phi$ takes integer values. The second term on the rhs of Eq. (9) implements the mesoscopic character of the phasefield model, whereby a finite interfacial width, $\epsilon$, appears affecting the step position. This term provides a surface tension contribution to the chemical potential and, in equilibrium, determines a stationary solution for the order parameter $\phi$ (for instance, for a double-well potential the stationary solution would be a hyperbolic tangent). Moreover, it will give rise to the diffusion term in the macroscopic model, Eq. (1). The third term on the rhs of Eq. (8) is akin to an external field $\mathbf{E}$ imposed on the order parameter. This term will give rise to step motion with a uniform velocity. Moreover, choosing appropriate $\sigma(\phi)$ and $\Gamma(\phi)$ functions will allow us to account for flux imbalance from both sides of the step, and will be responsible for the dynamic instability when the system is driven out of equilibrium.

In the following we will show how the macroscopic model is obtained from the phase-field model by an
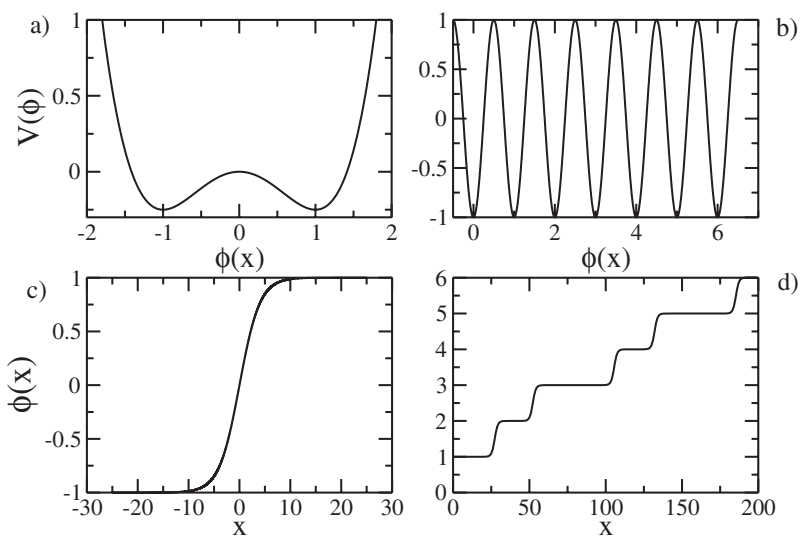

FIG. 1. Terrace potentials and phase-field solutions. (a) Bistable potential, Eq. (11). (b) Multistable periodic potential, Eq. (10) with $a=1$. (c) Numerical solution of Eq. (8) in one dimension using $V(\phi)$ as in (a). (d) Numerical solution of Eq. (8) in one dimension using $V(\phi)$ as in (b).

asymptotic expansion in the sharp interface limit $\epsilon \rightarrow 0$. At the different orders of this asymptotic expansion, we will recover from Eq. (8) both the dynamic equation for the bulk and the boundary conditions of the macroscopic model. This procedure will allow us to determine necessary conditions on $\Gamma(\phi)$ and $\sigma(\phi)$ (specifically, parity properties in terms of their arguments) in order to achieve such convergence. Apart from these requirements, we will carry out the asymptotic analysis for as general $\Gamma(\phi)$ and $\sigma(\phi)$ as possible. Finally, in our numerical integration of the phase-field model we will take explicit expressions of these functions.

For the sake of simplicity, we will concentrate in a single step separating two terraces. In such a case, one can replace the potential (10) by a double-well potential. Typically,

$$
V(\phi)=\frac{\phi^{4}}{4}-\frac{\phi^{2}}{2} .
$$

In this case, the lattice spacing is 2 (in nondimensional units), and the corrections to the constant values are signed, i.e., $\epsilon=A \widetilde{\epsilon}$ where $\tilde{\epsilon}$ is the interfacial width. Moreover, the phase field has the following asymptotic values:

$$
\lim _{z \rightarrow \pm \infty} \phi= \pm 1
$$

Figure 1 shows schematically the different alternatives for the phase-field potential and the stationary solutions corresponding to each choice. As we will show in Sec. III, in order to connect the macroscopic physical field $c_{k}$ to the phase field, $\phi$, we must perform the following change of variable:

$$
2 \Omega\left(c_{k}-c_{e q, 0}\right)=A(-A+\phi) .
$$

Equation (13) allows us to map the physical concentration field (which ranges from 0 to $c_{e q, 0}$ ) to the phase-field whose variation is given by Eq. (12). For the double-well potential given by Eq. (11) we take $A=\operatorname{sgn}[\phi]= \pm 1$. 


\section{ASYMPTOTIC EXPANSION}

We introduce curvilinear coordinates $(u, s)$, where $u$ is the normal distance to the interface and $s$ is the arclength (following the standard procedure, see Ref. [16] for details). The inner and outer expansion for a generic variable are written, respectively, as

$$
\begin{gathered}
\Phi=\Phi_{0}(w, s ; t)+\epsilon \Phi_{1}(w, s ; t)+\cdots, \\
\widetilde{\Phi}=\widetilde{\Phi}_{0}(u, s ; t)+\epsilon \widetilde{\Phi}_{1}(u, s ; t)+\cdots,
\end{gathered}
$$

where we have introduced the stretched normal coordinate for the inner region, $w=u / \epsilon$, and will use tilde for functions in the outer region.

The matching conditions are

$$
\begin{gathered}
\lim _{w \rightarrow \pm \infty} \Phi_{i}=\lim _{u \rightarrow 0^{ \pm}} \tilde{\Phi}_{i}, \\
\lim _{w \rightarrow \pm \infty} \Phi_{i+1, w}=\lim _{u \rightarrow 0^{ \pm}} \widetilde{\Phi}_{i, u},
\end{gathered}
$$

where $\Phi_{i, w}$ indicates the derivative in the direction of the coordinate $w$.

\section{A. Outer expansion}

\section{Order $\left(\epsilon^{0}\right)$}

From Eq. (8), at this order we obtain the dynamic equation

$$
\partial_{t} \widetilde{\phi}_{0}=\frac{A-\widetilde{\phi}_{0}}{\tau}+\nabla\left(\widetilde{\Gamma}_{0} \nabla \tilde{\mu}_{0}\right)-\mathbf{E} \cdot \nabla \sigma\left(\widetilde{\phi}_{0}\right),
$$

where $\widetilde{\Gamma}_{0} \equiv \Gamma\left(\widetilde{\phi}_{0}\right)$. Equation (9) gives

$$
\tilde{\mu}_{0}=-\widetilde{\phi}_{0}+\widetilde{\phi}_{0}^{3}
$$

From Eq. (12), we obtain $\tilde{\mu}_{0}=0$ and $A=\widetilde{\phi}_{0}$. Thus, at this lowest order the phase field takes constant values on the terraces sufficiently far from the step.

\section{Order $\left(\epsilon^{1}\right)$}

The dynamic equation at this order is

$$
\partial_{t} \widetilde{\phi}_{1}=-\frac{\widetilde{\phi}_{1}}{\tau}+\widetilde{\Gamma}_{0} \nabla^{2} \widetilde{\mu}_{1}-\sigma_{0}^{\prime} \mathbf{E} \cdot \nabla \widetilde{\phi}_{1},
$$

where $\sigma_{0}^{\prime} \equiv \sigma_{\phi}\left(\phi_{0}\right)$, and $V$ is related to $\mathbf{E}=E \hat{\mathbf{z}}$ and $\sigma$ by

$$
V \equiv \sigma_{0}^{\prime} E=V_{n}^{0} \hat{z}
$$

In order to obtain the same value of $V$ on the two sides of the terrace, we obtain as a necessary condition that $\sigma$ be an odd function of its argument.

At this order, Eq. (9) gives

$$
\tilde{\mu}_{1}=-\widetilde{\phi}_{1}+3 \widetilde{\phi}_{0}^{2} \widetilde{\phi}_{1}=2 \widetilde{\phi}_{1} .
$$

Using this result, Eq. (21) and Eq. (13) in Eq. (20), we recover the macroscopic diffusion equation, Eq. (1), with
$D \equiv 2 \widetilde{\Gamma}_{0}$, Eq. (22) thus allowing to relate $\widetilde{\Gamma}_{0}$ with a parameter of the macroscopic model. Likewise, Eq. (21) relates the external field $\mathbf{E}$ with the average step velocity, $V$, as anticipated.

\section{B. Inner expansion}

We now proceed to write Eq. (8) in the inner region,

$$
\begin{aligned}
\partial_{t} \phi-\frac{v}{\epsilon} \phi_{w}= & \frac{A-\phi}{\tau}+\frac{1}{\epsilon^{2}}\left(\Gamma \mu_{w}\right)_{w}-\frac{1}{\epsilon} \Gamma \kappa \mu_{w}-w \kappa^{2} \Gamma \mu_{w} \\
& +\left(\Gamma \mu_{s}\right)_{s}-\frac{1}{\epsilon} E_{w} \sigma^{\prime} \phi_{w}-E_{s} \sigma^{\prime} \phi_{s},
\end{aligned}
$$

where $E_{w}$ and $E_{s}$ are, respectively, the normal and tangential components of vector $\mathbf{E}$, and $v=v_{0}+\epsilon v_{1}+\cdots$ is the normal local velocity.

Equation (9) reads in this region as

$$
\frac{1}{\epsilon^{2}} \mu=\frac{1}{\epsilon^{2}}\left(-\phi+\phi^{3}-\phi_{w w}\right)+\frac{1}{\epsilon} \kappa \phi_{w}+w \kappa^{2} \phi_{w}-\phi_{s s} .
$$

$$
\text { 1. } \operatorname{Order}\left(\epsilon^{-2}\right)
$$

At this order, Eq. (23) becomes

$$
0=\left(\Gamma_{0} \mu_{0, w}\right)_{w},
$$

where $\Gamma_{0} \equiv \Gamma\left(\phi_{0}\right)$. Integrating twice we arrive at

$$
\mu_{0}=\int_{0}^{w} d w^{\prime} \frac{B}{\Gamma_{0}}+C,
$$

where $B$ and $C$ are integration constants. By using the matching conditions, we conclude that $B=C=0$ and then $\mu_{0}=0$, where

$$
\mu_{0}=-\phi_{0}+\phi_{0}^{3}-\phi_{0, w w} .
$$

The solution $\mu_{0}=0$ implies that $\phi_{0}$ is indeed a hyperbolic tangent,

$$
\phi_{0}=\tanh (w / \sqrt{2})
$$

from which $\phi_{0, w}$ provides a nontrivial eigenfunction (a Goldstone mode, having zero eigenvalue) for the differential operator

$$
\mathcal{L} \equiv-1+3 \phi_{0}^{2}-\partial_{w}^{2}
$$

\section{2. $\operatorname{Order}\left(\epsilon^{-1}\right)$}

At this order Eq. (23) reads as

$$
-v_{0} \phi_{0, w}=\left(\Gamma_{0} \mu_{1, w}\right)_{w}-E_{w} \sigma_{0}^{\prime} \phi_{0, w} .
$$

By integrating with respect to $w$ we obtain

$$
-v_{0} \phi_{0}=\Gamma_{0} \mu_{1, w}-E_{w} \sigma_{0}+T,
$$

where $T$ is the integration constant. Then, $\mu_{1, w}$ can be written as 


$$
\mu_{1, w}=-v_{0} \frac{\phi_{0}}{\Gamma_{0}}+E_{w} \frac{\sigma_{0}}{\Gamma_{0}}+\frac{T}{\Gamma_{0}} .
$$

Using the asymptotic matching and taking into account that $\tilde{\mu}_{0}=0$, we obtain

$$
\begin{gathered}
0=-v_{0} \frac{1}{\widetilde{\Gamma}_{0}}+E_{w} \frac{\sigma(+1)}{\widetilde{\Gamma}_{0}}+\frac{T}{\widetilde{\Gamma}_{0}}, \\
0=v_{0} \frac{1}{\widetilde{\Gamma}_{0}}+E_{w} \frac{\sigma(-1)}{\widetilde{\Gamma}_{0}}+\frac{T}{\widetilde{\Gamma}_{0}} .
\end{gathered}
$$

Given that $\sigma$ is an odd function, by adding the two previous equations we obtain $T=0$. By subtraction we obtain

$$
v_{0}=E_{w} \sigma(+1)=V_{n}
$$

Integrating again with respect to $w$ in Eq. (32) we obtain

$$
\mu_{1}=-v_{0} \int_{0}^{w} d w^{\prime} \frac{\phi_{0}}{\Gamma_{0}}+E_{w} \int_{0}^{w} d w^{\prime} \frac{\sigma_{0}}{\Gamma_{0}}+S
$$

where $S$ is a new integration constant.

In the same way, by integrating Eq. (24) we obtain

$$
\mu_{1}=-\phi_{1}+3 \phi_{0}^{2} \phi_{1}-\phi_{1, w w}+\kappa \phi_{0, w}
$$

and

$$
\mathcal{L} \phi_{1}=\mu_{1}-\kappa \phi_{0, w}
$$

The solvability condition for this nonhomogeneous equation implies

$$
0=\int_{-\infty}^{\infty} d w \mu_{1} \phi_{0, w}-\kappa \int_{-\infty}^{\infty} d w\left(\phi_{0, w}\right)^{2}
$$

and

$$
S=\frac{\gamma \kappa}{2}+\frac{v_{0}}{2} \alpha-\frac{E_{w}}{2} \delta
$$

where

$$
\begin{gathered}
\gamma=\int_{-\infty}^{\infty} d w\left(\phi_{0, w}\right)^{2}, \\
\alpha=\int_{-\infty}^{\infty} d w \int_{0}^{w} d w^{\prime} \frac{\phi_{0} \phi_{0, w}}{\Gamma_{0}}, \\
\delta=\int_{-\infty}^{\infty} d w \int_{0}^{w} d w^{\prime} \frac{\sigma_{0} \phi_{0, w}}{\Gamma_{0}} .
\end{gathered}
$$

Introducing now $S$ in Eq. (36) and applying the matching condition with the outer variables, we obtain

$$
2 \widetilde{\phi}_{1}^{+}=-v_{0} \int_{0}^{\infty} d w^{\prime} \frac{\phi_{0}}{\Gamma_{0}}+E_{w} \int_{0}^{\infty} d w^{\prime} \frac{\sigma_{0}}{\Gamma_{0}}+\frac{\gamma \kappa}{2}+\frac{v_{0}}{2} \alpha-\frac{E_{w}}{2} \delta,
$$

$2 \widetilde{\phi}_{1}^{-}=-v_{0} \int_{0}^{-\infty} d w^{\prime} \frac{\phi_{0}}{\Gamma_{0}}+E_{w} \int_{0}^{-\infty} d w^{\prime} \frac{\sigma_{0}}{\Gamma_{0}}+\frac{\gamma \kappa}{2}+\frac{v_{0}}{2} \alpha-\frac{E_{w}}{2} \delta$,

where we have used that $\tilde{\mu}_{1}=2 \widetilde{\phi}_{1}$. Now, we define two new constants $k_{ \pm}$as

$$
k_{ \pm}^{-1}=-\int_{0}^{ \pm \infty} d w \frac{\phi_{0}}{\Gamma_{0}}+\frac{1}{\sigma(+1)} \int_{0}^{ \pm \infty} d w \frac{\sigma_{0}}{\Gamma_{0}}+\frac{\alpha}{2}-\frac{\delta}{2 \sigma(+1)} .
$$

Note that the asymmetry in the attachment is due to the mobility function, $\Gamma_{0}=\Gamma\left(\phi_{0}\right)$ and not to the value of the external drive $\mathbf{E}$.

Adding Eqs. (44) and (45) we obtain

$$
v_{0}=k_{+}\left(\widetilde{\phi}_{1}^{+}-\frac{\gamma}{4} \kappa\right)+k_{-}\left(\widetilde{\phi}_{1}^{-}-\frac{\gamma}{4} \kappa\right) .
$$

This equation becomes the macroscopic equation for the step velocity, Eq. (2), taking into account that

$$
\widetilde{\phi}_{1}^{ \pm}=2 \Omega\left(c_{ \pm}-c_{e q}\right),
$$

and identifying $\nu_{ \pm}=2 \Omega k_{ \pm}$and $\Gamma=\frac{\gamma}{8 \Omega}$ (note that $\Omega \Gamma \equiv d_{0}$ is the so-called capillarity length).

\section{3. $\operatorname{Order}\left(\epsilon^{0}\right)$}

The last macroscopic equation, the continuity equation, Eq. (4), can be retrieved already at this order. Equation (23) can be written as

$$
\begin{aligned}
-v_{1} \phi_{0, w}-v_{0} \phi_{1, w}= & \frac{A-\phi_{0}}{\tau}+\left(\Gamma_{0} \mu_{2, w}\right)_{w}+\left(\Gamma_{0}^{\prime} \phi_{1} \mu_{1, w}\right)_{w} \\
& -\kappa \Gamma_{0} \mu_{1, w}-E_{w} \sigma_{0}^{\prime} \phi_{1, w}-E_{w} \sigma_{0}^{\prime \prime} \phi_{1} \phi_{0, w} .
\end{aligned}
$$

Again integrating with respect to $w$,

$$
\begin{aligned}
-v_{1} \phi_{0}-v_{0} \phi_{1}= & \int_{0}^{w} d w\left(\frac{A-\phi_{0}}{\tau}\right)+\Gamma_{0} \mu_{2, w}+\Gamma_{0}^{\prime} \phi_{1} \mu_{1, w} \\
& -\kappa \Gamma_{0} \mu_{1}-E_{w} \sigma_{0}^{\prime} \phi_{1} .
\end{aligned}
$$

By imposing the matching condition, we obtain two equations, one for each phase (terrace)

$$
\begin{aligned}
-v_{1}-v_{0} \widetilde{\phi}_{1}^{+}= & \int_{0}^{\infty} d w\left(\frac{A-\phi_{0}}{\tau}\right)+\widetilde{\Gamma}_{0} \widetilde{\mu}_{1, u}^{+} \\
& -\kappa \widetilde{\Gamma}_{0} \tilde{\mu}_{1}^{+}-E_{w} \sigma^{\prime}(+1) \widetilde{\phi}_{1}^{+}, \\
v_{1}-v_{0} \widetilde{\phi}_{1}^{-}= & \int_{0}^{-\infty} d w\left(\frac{A-\phi_{0}}{\tau}\right)+\widetilde{\Gamma}_{0} \tilde{\mu}_{1, u}^{-} \\
& -\kappa \widetilde{\Gamma}_{0} \tilde{\mu}_{1}^{-}-E_{w} \sigma^{\prime}(-1) \widetilde{\phi}_{1}^{-} .
\end{aligned}
$$

Taking into account the expressions for $A$ [Eq. (13)], and $\phi_{0}$, [Eq. (28)], and subtracting Eqs. (51) and (52), we obtain 


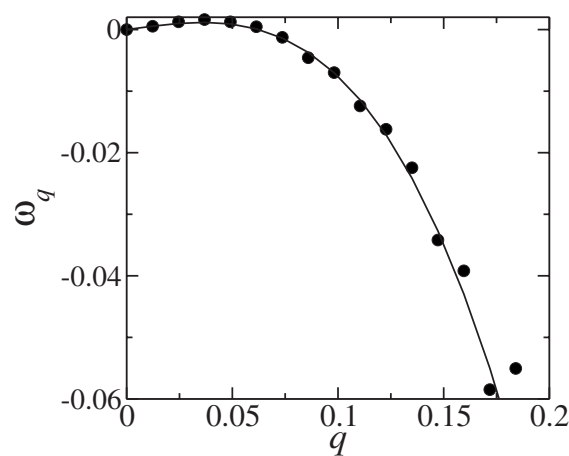

FIG. 2. Linear dispersion relation for the symmetric attachment case, $\Gamma(\phi)=\Gamma_{a}=1$. The circles provide the results of the numerical integration and the solid line is a fit to the linear dispersion relation (55) [34]. Other parameters are $\Delta x=1, \Delta t=0.005$, system size $L_{x}$ $\times L_{y}=512 \times 512, \epsilon=0.5$, and $\tau=100$. All units are arbitrary.

$$
2 v_{1}=\Gamma_{0}\left(\widetilde{\mu}_{1, u}^{-}-\tilde{\mu}_{1, u}^{+}\right)+\Gamma_{0} \kappa\left(\tilde{\mu}_{1}^{-}-\tilde{\mu}_{1}^{+}\right)+V_{n}^{0}\left(\widetilde{\phi}_{1}^{-}-\widetilde{\phi}_{1}^{+}\right) .
$$

We can recognize here Eq. (4), once we take into account that the first two terms on the right-hand side of (53) yield the normal derivative of $c$, and that $v_{1}=V_{n}^{l}$ after the change of variables, Eq. (48), as above.

\section{NUMERICAL INTEGRATION}

In this section we briefly present some results from the numerical integration of Eq. (8) in order to check the validity of the approximations made in the preceding sections.

We have used a fourth-order Runge-Kutta integration scheme for the time evolution (with time step $\Delta t$ ), and a discretization of spatial derivatives using nearest-neighbor finite differences (with lattice spacing $\Delta x$ ). In all the computations we have used

$$
-\sigma^{\prime}(\phi)=1+0.1 \phi^{2}\left(-6+5 \phi^{2}\right) \text {. }
$$

This choice guarantees that the mean velocity of the front is nonvanishing.

Besides, we have performed a couple of numerical experiments to check the qualitative and quantitative validity of the sharp-interface limit exposed in Sec. III. The first set of numerical data models the case of an interface separating two phases with equal probabilities of attachment (namely, $\nu_{+}$ $\left.=\nu_{-}\right)$with desorption $(\tau=100)$. In this symmetric case, some analytical results can be obtained [34] so we will use them to check the validity of our approximation. Then, the second set of numerical data deals with the case of asymmetric attachment, also with desorption $(\tau=10)$. Although in principle the matching of the parameters can be also done in this case, the function $\Gamma$ must be chosen appropriately.

In Fig. 2 we show the dispersion relation $\left(\omega_{q}\right)$ in the case of symmetric attachment [in the phase-field model this means that $\Gamma(\phi)=\Gamma_{0}=$ constant $]$, as obtained numerically. Analytically, it can be shown that in this case [34]

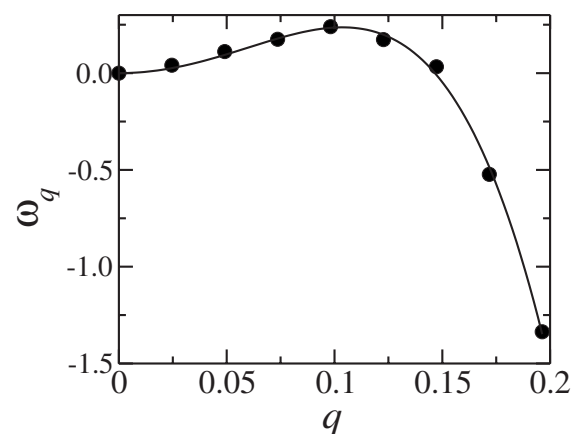

FIG. 3. Linear dispersion relation for the asymmetric attachment case $\left[\Gamma(\phi)=\Gamma_{a}+\Gamma_{s}(\phi+\lambda)\left(1-\phi^{2}\right)\right]$. The circles provide the results of the numerical integration and the solid line is a fit to the linear dispersion relation (7) [8]. Parameters are $\Delta x=1, \Delta t=0.005$, system size $L_{x} \times L_{y}=512 \times 512, \epsilon=0.5$, and $\tau=10$. All units are arbitrary.

$\omega_{q}=\frac{D}{x_{s}^{2}}\left\{\Omega\left(F \tau-c_{e q}^{0}\right)\left[\sqrt{1+\left(q x_{s}\right)^{2}}-1\right]-d_{0} x_{s} c_{e q}^{0} q^{2} \sqrt{1+\left(q x_{s}\right)^{2}}\right\}$,

with $x_{s}=(D \tau)^{1 / 2}$

In order to obtain $\omega_{q}$ numerically, we impose a sinusoidal initial condition for the zero level set of the phase field, with spatial frequency $q$. The system is integrated and the curve $h(x, t)$ related to the zero level set is assumed to grow as $h(x, t) \sim \exp \left(\omega_{q} t\right)$. In Fig. 2, the dots are obtained by this method from numerics and the solid line is a fit to Eq. (55). Note that the change of variables given in Eq. (13) provides two fitting parameters $\left(\Omega\right.$ and $\left.c_{e q}\right)$ that can be obtained.

In the case of asymmetric attachment with desorption, we have just performed the comparison qualitatively. Thus, in Fig. 3 we show the dispersion relation $\left(\omega_{q}\right)$ where the asymmetry has been introduced through a functional dependence of $\Gamma$ on the phase field. We have chosen (for simplicity)

$$
\Gamma(\phi)=\Gamma_{a}+\Gamma_{s}(\phi+\lambda)\left(1-\phi^{2}\right),
$$

with $\Gamma_{a}=\Gamma_{s}=1$, and $\lambda=0.5$. This form ensures that the macroscopic diffusivity, $D$ is equal in both phases but also provides the asymmetry in the coefficients $\nu_{ \pm}$through Eq. (46). The circles stand for numerical data and the solid line for a fit to Eq. (7).

As can be seen the evolution of an initially disordered step we reproduce the linear dispersion relation (7), as expected for the full macroscopic equations (1)-(4) [8], similar to other phase-field formulations [22].

In Fig. 4 we show the global interface width defined as the root mean square of the step position. The behavior is that predicted by the Kuramoto-Sivashinsky equation: An initial transient followed by an exponential regime, and finally a scaling regime with exponents that are compatible with those of the Kardar-Parisi-Zhang universality class, only after a long transient associated with the corresponding linear theory [35]. Analogously, in Fig. 5 we show the power spectral density for the same system parameters. Note the -4 slope at high frequencies which is, again, compatible with the predicted (noisy) Kuramoto-Sivashinsky behavior [36]. 


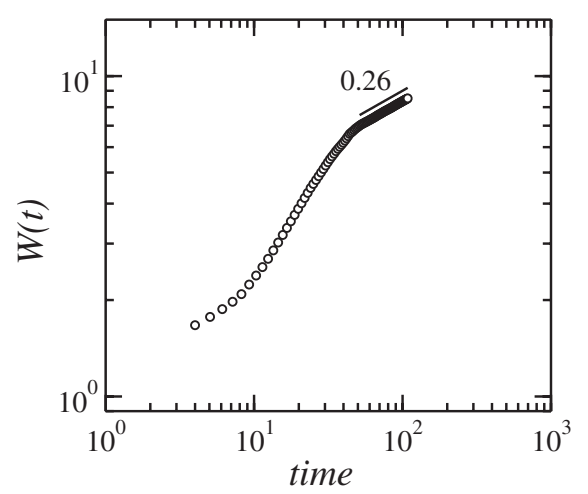

FIG. 4. Global interface width (root mean square of the interface height), with $\Delta x=1, \Delta t=0.005$, system size $L_{x} \times L_{y}=512 \times 512, \epsilon$ $=0.5$, and $\tau=10$. The straight line is a guide to the eye with slope 0.26 (in agreement with the Kuramoto-Sivashinsky equation for small systems [35]). All units are arbitrary.

\section{DISCUSSION AND CONCLUSIONS}

In this paper we have introduced a one-field phase-field model that reproduces the main physical description of step dynamics in the step-flow regime, as provided by the BCF model. This type of stepped system has traditionally been studied (within the phase-field formalism) with, at least, two coupled fields: One field for the physical adatom concentration on the terraces and another order parameter-type field tracking the step position. We have shown the equivalence between the moving boundary problem and our simple formulation by means of asymptotic analysis and numerical integration.

Our present contribution can be relevant not only from the point of view of economy of equations but also its capability to be generalized to more complex or specialized problems. Moreover, it allows us to explore nonlinear regimes and parameter conditions that may lay beyond usual asymptotic approximations, common to effective interfacial theories.

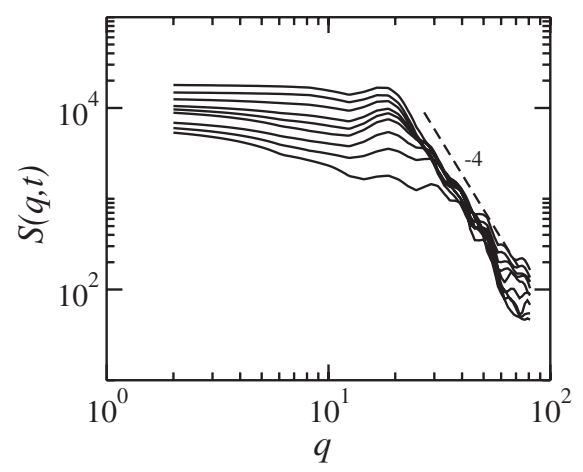

FIG. 5. Power spectral density of the step position for different times with $\Delta x=1, \Delta t=0.005$, system size $L_{x} \times L_{y}=512 \times 512, \epsilon$ $=0.5$, and $\tau=10$. The dashed line is guide to the eye with slope -4 (in agreement with the Kuramoto-Sivashinsky equation). All units are arbitrary.

Among natural generalizations of our model we wish to mention electromigration $[37,38]$ that might be implemented in our framework [by appropriately tuning the $\mathbf{E}$ field in (8) to accommodate the additional advective term that appears in (1) under such physical conditions], KESE instabilities [14] (which are Ehrlich-Schoewbel instabilities located at the step kinks and not between terraces); the effect of pinning due to impurities (for instance, by introducing a quenched noise contribution to function $\Gamma$ ), or the role of thermal fluctuations (relevant at the nanoscale) by simply adding the proper noise terms in Eq. (8).

\section{ACKNOWLEDGMENTS}

We acknowledge collaborations and interactions with $\mathrm{T}$. Ala-Nissila, K. R. Elder, J. Heinonen, I. T. Koponen, M. Rusanen, and P. Cervera at various stages of this work. The present work has been partially supported by MEC (Spain) Grants No. FIS2006-12253-C06-01, No. FIS2006-12253C06-05, and No. FIS2006-12253-C06-06, UC3M/CAM (Spain) Grant No. UC3M-FI-05-007, and CAM (Spain) Grant No. S-0505/ESP-0158.
[1] See, e.g., N. Néel, T. Maroutian, L. Douillard, and H.-J. Ernst, J. Phys.: Condens. Matter 15, S3227 (2003), and other contributions in the same issue.

[2] A. Pimpinelli and J. Villain, Physics of Crystal Growth (Cambridge University Press, Cambridge, 1998).

[3] T. Michely and J. Krug, Islands, Mounds, and Atoms: Patterns and Processes in Crystal Growth Far from Equilibrium (Springer, Heidelberg, 2004).

[4] H. Jeong and E. Williams, Surf. Sci. Rep. 34, 171 (1999).

[5] D. Margetis, Phys. Rev. B 76, 193403 (2007).

[6] W. Burton, N. Cabrera, and F. Frank, Philos. Trans. R. Soc. London, Ser. A 243, 299 (1951).

[7] P. Politi et al., Phys. Rep. 324, 271 (2000).

[8] G. S. Bales and A. Zangwill, Phys. Rev. B 41, 5500 (1990).

[9] O. Pierre-Louis and C. Misbah, Phys. Rev. B 58, 2259 (1998).

[10] O. Pierre-Louis, C. Misbah, Y. Saito, J. Krug, and P. Politi,
Phys. Rev. Lett. 80, 4221 (1998).

[11] G. Danker, O. Pierre-Louis, K. Kassner, and C. Misbah, Phys. Rev. Lett. 93, 185504 (2004).

[12] J. Krug, V. Tonchev, S. Stoyanov, and A. Pimpinelli, Phys. Rev. B 71, 045412 (2005).

[13] J. Tersoff, Y. H. Phang, Z. Zhang, and M. G. Lagally, Phys. Rev. Lett. 75, 2730 (1995).

[14] M. Rusanen, I. T. Koponen, J. Heinonen, and T. Ala-Nissila, Phys. Rev. Lett. 86, 5317 (2001).

[15] J. Krug, in Collective Dynamics of Nonlinear and Disordered Systems, edited by G. Randons, P. Häusler, and W. Just (Springer, Berlin, 2004), pp. 5-38.

[16] R. González-Cinca et al., in Advances in Condensed Matter and Statistical Physics, edited by E. Korutcheva and R. Cuerno (Nova Science, New York, 2004), pp. 203-236.

[17] See, e.g., W. J. Boettinger, J. A. Warren, C. Beckermann, and 
A. Karma, Annu. Rev. Mater. Res. 32, 163 (2002), and other contributions in the same volume.

[18] G. Lu and E. Kaxiras, in Handbook of Theoretical and Computational Nanotechnology, edited by M. Rieth and W. Schommers (American Scientific, Valencia, 2005), pp. 1-33.

[19] F. Liu and H. Metiu, Phys. Rev. E 49, 2601 (1994).

[20] A. Karma and M. Plapp, Phys. Rev. Lett. 81, 4444 (1998).

[21] A. Rätz and A. Voigt, Appl. Anal. 83, 1015 (2004).

[22] O. Pierre-Louis, Phys. Rev. E 68, 021604 (2003).

[23] F. Otto et al., Nonlinearity 17, 477 (2004).

[24] J.-Y. Kim, D.-H. Yeon, P.-R. Cha, and J.-K. Yoon, Mater. Sci Forum 475-479, 3181 (2005).

[25] Y.-M. Yu and B.-G. Liu, Phys. Rev. B 73, 035416 (2006).

[26] P. C. Hohenberg and B. I. Halperin, Rev. Mod. Phys. 49, 435 (1977).

[27] J. Soriano, J. J. Ramasco, M. A. Rodriguez, A. HernandezMachado, and J. Ortin, Phys. Rev. Lett. 89, 026102 (2002).

[28] A. Hernández-Machado, A. M. Lacasta, E. Mayoral, and E. C. Poiré, Phys. Rev. E 68, 046310 (2003).
[29] M. Castro, Phys. Rev. B 67, 035412 (2003).

[30] K. R. Elder, M. Grant, N. Provatas, and J. M. Kosterlitz, Phys. Rev. E 64, 021604 (2001).

[31] Y. Kuramoto, Chemical Osillation, Waves and Turbulence (Springer, Heidelberg, 1984).

[32] I. Bena, C. Misbah, and A. Valance, Phys. Rev. B 47, 7408 (1993).

[33] T. Frisch and A. Verga, Physica D 235, 15 (2007).

[34] A. Karma and C. Misbah, Phys. Rev. Lett. 71, 3810 (1993).

[35] K. Sneppen, J. Krug, M. H. Jensen, C. Jayaprakash, and T. Bohr, Phys. Rev. A 46, R7351 (1992).

[36] R. Cuerno, H. A. Makse, S. Tomassone, S. T. Harrington, and H. E. Stanley, Phys. Rev. Lett. 75, 4464 (1995).

[37] J. Chang, O. Pierre-Louis, and C. Misbah, Phys. Rev. Lett. 96, 195901 (2006).

[38] M. Dufay, J.-M. Debierre, and T. Frisch, Phys. Rev. B 75, 045413 (2007). 